\title{
Fault tolerant control of uav with wing layout based on control allocation
}

\author{
Chen $\mathrm{Jie}^{1^{*}}$, LIN Jianxin ${ }^{2}$ \\ ${ }^{1}$ Combat college, naval aviation university, yantai, 264001, Shandong, China \\ ${ }^{2}$ Army 92692 Fujian Zhanjiang 524000.)
}

\begin{abstract}
As the flying wing layout unmanned aerial vehicle (uav) extensive research and task environment increasingly complex, Yu Feiyi layout unmanned aerial vehicle (uav) for fault tolerant control gradually become the main technical means of the flight control, using the established mathematical model of the flying wing uav longitudinal layout setting the actuator failure effect, is in the nature of adaptive control allocation fault-tolerant algorithm is given, and MATLAB/simulink simulation is carried out for uav longitudinal motion, realize the rapid and stable, the control command and response to complete the nonlinear fault-tolerant control of flying wing uavs.
\end{abstract}

\section{Introduction}

In consideration of the changing aerodynamic configuration, the flying-wing UAV (unmanned aerial vehicle) is equipped with the structure of multiple rudder surfaces, which gives rise to a variety of combinations of various rudder surfaces. If the torque and the angular acceleration are respectively projected into the three axes of the airframe structure's axle system, and then the control allocation algorithm is used to process the effective and rational allocation of multiple rudder surfaces in performance control, not only the UAV can be allowed to adapt to different flight conditions, but also loss in various aspects can be reduced, which will significantly increase the control efficiency and the fault-tolerance performance of the UAV with the structure of multiple rudder surfaces.

The fault-tolerant algorithm for the control allocation of the flying-wing UAV, designed in this section, is based on the configuration of the flying-wing UAV in Figure 1, applied for the strong self-adaptability of the design algorithm and its strong fault-tolerant control ability if the rudder surface fails to function. The basic principle of the distribution algorithm is that the pseudo-control quantity is output after the system input instruction passes through the system control module, and the pseudo-control quantity is then output to each rudder surface as the position instruction after it passes through the control distribution processor. Finally, the rudder surface tracks these position instructions to obtain the torque and angular acceleration which are required by the system in real time.

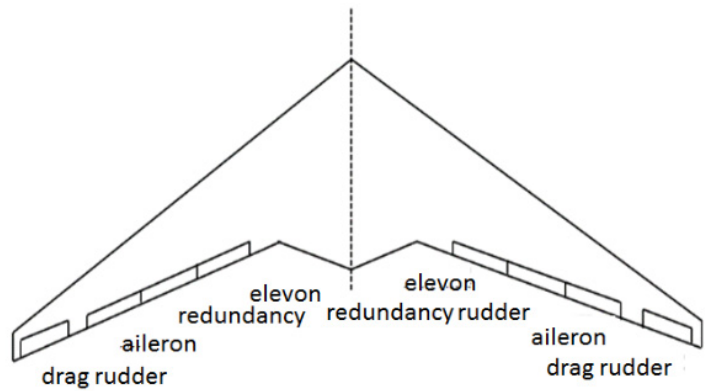

Figure 1 The Sample Graph of the Flying-Wing UAV

Under the malfunctioning condition, the distribution control system uses the aerodynamic redundancy of the rudder to actively fault-tolerate such malfunction as the failure of the elevon to ensure the safety and reliability of the UAV. After the adaptive control allocation and then the reintegration, the purpose of the self-correcting system is achieved, so that the UAV reduces its requirement for the hardware redundancy of the control system so as to effectively improve the survivability of the UAV.

In the following parts, the problem is transformed into one of the continuous multivariable optimizations, and a new multi-control surface reconstruction distribution method is proposed by using the evolution algorithm based on the distribution to obtain the global optimal solution.

The comprehensive utilization and combined control of the rudder surface can fully explore the potential of each rudder surface and the airframe structure, and at the same time reduce the cost and price, it can still greatly improve the flight performance of the UAV. 


\section{Describing the Issue of Control Distribution}

\subsection{Analyzing the Characteristic of Multiple Rudder Surfaces in the Distribution of the Flying Wing}

Except that the drag rudder is of the split mode, the other rudder surfaces impose an aerodynamic influence on the UAV according to the same principle, although with a different efficiency of the rudder surface. From the perspective of control, any control function of the UAV is a control redistribution based on the original control law.

Although each rudder surface of the flying-wing UAV is similar in its function, the actual function of the rudder surface can be set artificially according to the change of aerodynamic data, which is conducive to the optimal allocation of rudder surface function. In this section, according to the efficiency of the elevon, the redundancy rudder and the aileron in controlling the rudder surface in the aerodynamic data, the functions of rudder surface are specified as shown in Table 1.

Table 1 Main control surfaces and functions of flying-wing UAV

\begin{tabular}{lll}
\hline \multicolumn{1}{c}{ Rudder Surface } & Main Functions & Other Functions \\
\hline Elevon & pitch control & lateral control, reinforcement, \\
& balancing & reinforcement, balancing \\
redundancy rudder & pitch or lateral control & pitch control, reinforcement, balancing \\
aileron & lateral control & reinforcement, balancing \\
rudder & course control & r \\
\hline
\end{tabular}

Table 1 shows the main functions and auxiliary functions of the flying-wing UAV's four pairs of rudder surfaces, among which the main functions of each rudder surface correspond to the relative efficiency in the control of rudder surface respectively. In the control distribution, the main functions of each rudder surface should be considered primarily, so that the rudder surface with relatively high control efficiency can play more of the auxiliary control role, and at the same time, the control distribution scheme with small total deflection and optimization should be put forward.

\subsection{Defining the Longitudinal Model and the Performance Function of the Flying-Wing UAV}

In this section, in view of the issue of the flying-wing fault-tolerant control, a self-adaptive tracking control method of backstepping compensation is put forward in consideration of the changed systematical dynamic performance on the prescribed performance bound, according to the flying-wing UAV's dynamic performance of the tracking system when in the malfunction of the rudder surface. This method combines the distribution features of the UAV's rudder surface, designs a control allocation scheme of the rudder surface, to avoid the longitudinal and latitudinal coupling caused by the malfunction of rudder surface, and ensures that the system can maintain stability and asymptotically track the given reference signal when the failure of control surface occurs.

As shown in Figure 1, the flying-wing UAV has no horizontal tail or vertical tail, and all its rudder surfaces are arranged on the trailing edge of the serrated wing, including the elevon, the redundancy rudder, the ailerons and the rudder. Among them, the deflection range of the elevon and of the rudder are respectively $\pm 25^{\circ}$ and $\pm 90^{\circ}$.

In the analysis, only the longitudinal control problem is considered. First, according to the full-state model of $\mathrm{UAV}$, the longitudinal motion equations is given as followed:

$$
\left\{\begin{aligned}
\dot{V}= & -\frac{1}{2 m} \rho V^{2} S\left[C_{M_{0}}+k\left(C_{l_{z}} \alpha+\frac{\bar{c}}{2 V} C_{D_{z}} q+\frac{\bar{c}}{2 V} C_{M_{z}} \dot{\alpha}\right)^{2}\right] \\
& -g(\cos \alpha \sin \theta-\sin \alpha \cos \theta)+\frac{1}{m} T_{\max } \cos \alpha u_{t} \\
\dot{\alpha}= & q-\frac{1}{2 m} \rho V S\left(C_{l_{z}} \alpha+\frac{\bar{c}}{2 V} C_{D_{z}} q+\frac{\bar{c}}{2 V} C_{M_{z}} \dot{\alpha}\right) \\
& +\frac{1}{V} g(\sin \alpha \sin \theta+\cos \alpha \cos \theta)-\frac{1}{m V} T_{\max } \sin \alpha u_{t} \\
\dot{\theta}= & q \\
\dot{q}= & \frac{1}{2 I_{y}} \rho V^{2} S \bar{c}\left(C_{M_{0}}+C_{l_{z}} \alpha+\frac{\bar{c}}{2 V} C_{D_{z}} q+\frac{\bar{c}}{2 V} C_{M_{z}} \dot{\alpha}\right) \\
& +\frac{1}{I_{y}} T_{\max } u_{t}+\frac{1}{2 I_{y}} \rho V^{2} S \bar{c} C_{M} \\
\dot{h}= & V \sin (\theta-\alpha)
\end{aligned}\right.
$$

In this equation, $T_{\max }$ is the maximum thrust of the UAV's engine, and $u_{t}$ is the throttle opening of the engine. Other variables are shown in Reference [11].

In the longitudinal nonlinear set of system equation (1), the input is defined as $\left[u_{t}, u_{e}\right]^{T}$, respectively referring to the throttle opening of the engine and the deflection angle of the two pairs of rudder surfaces of lifting functions (the aileron and the redundancy rudder), namely, the input signal of the engine is

$$
u_{t}=\left(u_{t 1}+u_{t 2}\right) \text {, }
$$

and the input signal of the elevon is

$$
u_{e}=\left(u_{1}+u_{1}^{\prime}+u_{2}+u_{2}^{\prime}\right)
$$

In this paper, the fault-tolerant control of the longitudinal elevator surface under the malfunction state is considered. In order to represent the malfunction of the 
rudder, according to the configuration of multiple rudders, $\lambda$ is defined as the proportional coefficient that represents the partial failure of a certain elevator surface in the system. Obviously, $\lambda \leq 1$. The relationship between $u_{e}^{\prime}$, the actual output of the elevator, and the input of the rudder's malfunction condition is defined as

$$
u_{e}^{\prime}=\lambda u_{e}(t)
$$

Compared with the pitch angle and the flight speed, the flight altitude is the slow variable of the longitudinal motion. In order to demonstrate the characteristics and effectiveness of the control method in this section, $y=\left[\begin{array}{ll}V & \theta\end{array}\right]^{T}$ is selected as the system output. In order to consider the physical limitations of the system actuator in terms of the control objective, the control law is set so that the system (1) can maintain the stable flight in the malfunction of the control surface (2), and the speed and the output of the pitch angle $y$ can track the reference signal with predetermined performance.

The tracking error of the system is set as:

$$
e_{i}(t)=y_{i}(t)-y_{r i}(t), i=1,2
$$

and a performance index function is defined as:

$$
\Gamma=\left\{\left(t, e_{i}\right) \in R_{t \geq 0} \times R \mid \chi_{i 0}^{-}(t)<e_{i}(t)<\chi_{i 0}^{+}(t)\right\}, i=1,2
$$

In this formula, $\chi_{i 0}^{-}(t)$ and $\chi_{i 0}^{+}(t)$ are smooth performance functions, and they satisfy the following requirements:

1) $\chi_{i 0}^{-}(t)$ and $\chi_{i 0}^{+}(t)$ and their second derivative is bounded;

2) $\lim _{t \rightarrow \infty} \chi_{i 0}^{-}(t)=\chi_{i}^{-}, \lim _{t \rightarrow \infty} \chi_{i 0}^{+}(t)=\chi_{i}^{+}$, and $\chi_{i}^{-}, \chi_{i}^{+}$are specified constants.

If $\chi_{i 0}^{-}(t)=-\zeta_{i} \tau_{i}(t), \chi_{i 0}^{+}(t)=\bar{\zeta}_{i} \tau_{i}(t)$, the performance index function $\Gamma$ will be

$$
-\zeta_{i} \tau_{i}(t)<e_{i}(t)<\bar{\zeta}_{i} \tau_{i}(t), \forall t \geq 0, \quad i=1,2
$$

In it, $0<\zeta_{i}, \bar{\zeta}_{i} \leq 1 ; \tau_{i}(t)$ is the system performance function that needs to be designed, and it is required to be the same smooth decreasing function, while $0<\lim _{t \rightarrow \infty} \tau_{i}(t)=\tau_{i \infty}<\tau_{i 0}$.

If $\tau_{i}(t)=\left(\tau_{i 0}-\tau_{i \infty}\right) e^{-a_{i} t}+\tau_{i \infty}, \quad \tau_{i \infty}, \quad \tau_{i \infty}$ and $a_{i}$ are the preset positive constants, $-\zeta_{i} \tau_{i \infty}$ represents the upper bound of the predetermined steady-state error, and $\bar{\zeta}_{i} \tau_{i \infty}$ represents the lower bound of the predetermined steady-state error. If $e_{i}(t)>0, \bar{\zeta}_{i} \tau_{i}(t)$ is the upper bound of the tracking error, the maximum overshoot is less than $\bar{\zeta}_{i} \tau_{i 0}$; If $e_{i}(t)<0,-\zeta_{i} \tau_{i}(t)$ is the lower bound of the tracking error, the maximum overshoot is less than $-\zeta_{i} \tau_{i 0}$.

If equation (4) is satisfied by $e_{i}(t)$ under the control effect, the output tracking error of the system is bounded and $-\zeta_{i} \tau_{i \infty}<\lim _{t \rightarrow \infty} e_{i}(t)<\bar{\zeta}_{i} \tau_{i \infty}$.

\section{Designing the Fault-Tolerant Control Law for Flying-Wing UAV}

\subsection{Transformation of the Performance Bounds of System Setting}

Design a strictly increasing function as $S\left(v_{i}\right), i=1,2$ to satisfy the requirements of

$$
\begin{aligned}
& -\zeta_{i}<S\left(v_{i}\right)<\bar{\zeta}_{i} \\
& \lim _{v_{i} \rightarrow \infty} S\left(v_{i}\right)=\bar{\zeta}_{i}, \lim _{v_{i} \rightarrow-\infty} S\left(v_{i}\right)=-\zeta_{i} \\
& S(0)=0
\end{aligned}
$$

In this thesis, $e_{i}(t)=\tau_{i}(t) S\left(v_{i}\right)$ is chosen to satisfy formula (4), and $S\left(v_{i}\right)$ is adopted in the following set:

$$
S\left(v_{i}\right)=\frac{\bar{\zeta} e^{\left(v_{i}+\gamma_{i}\right)}-\zeta_{i} e^{-\left(v_{i}+\gamma_{i}\right)}}{e^{\left(v_{i}+\gamma_{i}\right)}+e^{-\left(v_{i}+\gamma_{i}\right)}}
$$

In this formula, $\gamma_{i}=\frac{\ln \frac{-\zeta_{i}}{\bar{\zeta}_{i}}}{2}$, and it can be concluded that $S\left(v_{i}\right)$ can satisfy condition (5), and it can be concluded from $e_{i}(t)=\tau_{i}(t) S\left(v_{i}\right)$ and formula (6) that:

$v_{i}=\frac{1}{2} \ln \left(\bar{\zeta}_{i} \frac{e_{i}(t)}{\tau_{i}(t)}+\bar{\zeta}_{i}\left(-\zeta_{i}\right)\right)-\frac{1}{2} \ln \left(\bar{\zeta}_{i}\left(-\zeta_{i}\right)-\left(-\zeta_{i}\right) \frac{e_{i}(t)}{\tau_{i}(t)}\right)$

If take the derivative of $v_{i}$, it can be obtained:

$$
\begin{aligned}
& \dot{v}_{1}=\frac{\partial S^{-1}}{\partial \frac{e_{1}(t)}{\tau_{1}(t)}\left(\frac{e_{1}(t)}{\tau_{1}(t)}\right)^{\prime}=S_{1}\left(\dot{V}-\dot{V}_{r}-\frac{e_{1} \dot{\tau}_{1}}{\tau_{1}}\right)} \\
& \dot{v}_{2}=\frac{\partial S^{-1}}{\partial \frac{e_{2}(t)}{\tau_{2}(t)}\left(\frac{e_{2}(t)}{\tau_{2}(t)}\right)^{\prime}=S_{2}\left(\dot{\theta}-\dot{\theta}_{r}-\frac{e_{2} \dot{\tau}_{2}}{\tau_{2}}\right)}
\end{aligned}
$$

In it, $S\left(v_{i}\right)=\frac{1}{2 \tau_{i}}\left[\frac{1}{\frac{e_{i}(t)}{\tau_{i}(t)}+\left(-\zeta_{i}\right)}-\frac{1}{\frac{e_{i}(t)}{\tau_{i}(t)}-\bar{\zeta}_{i}}\right]$. System

will go through the transformation: 


$$
\begin{aligned}
& \dot{v}_{1}=S_{1}\left(f_{1}+\frac{1}{m} T_{\max } \cos \alpha u_{t}-\dot{V}_{r}-\frac{e_{1} \dot{\tau}_{1}}{\tau_{1}}\right) \\
& \dot{v}_{2}=S_{2}\left(q-\dot{\theta}_{r}-\frac{e_{2} \dot{\tau}_{2}}{\tau_{2}}\right) \\
& \dot{q}=f_{2}+\frac{1}{2 I_{y}} \rho V^{2} S c C_{M}
\end{aligned}
$$

In it,

$$
\begin{aligned}
& f_{1}=-\frac{1}{2 m} \rho V^{2} S\left[C_{M_{0}}+k\left(C_{l_{z}} \alpha+\frac{\bar{c}}{2 V} C_{D_{z}} q+\frac{\bar{c}}{2 V} C_{M_{z}} \dot{\alpha}\right)^{2}\right]-g(\cos \alpha \sin \theta-\sin \alpha \cos \theta) \\
& f_{2}=q-\frac{1}{2 m} \rho V S\left(C_{l_{z}} \alpha+\frac{\bar{c}}{2 V} C_{D_{z}} q+\frac{\bar{c}}{2 V} C_{M_{z}} \dot{\alpha}\right)+\frac{1}{V} g(\sin \alpha \sin \theta+\cos \alpha \cos \theta) \quad \text { If }
\end{aligned}
$$

If $v_{1}$ is set as:

$$
z_{11}=v_{1}
$$

Take the derivative of formula (10), and it can be gotten:

$$
z_{11}=S_{1}\left(f_{1}+\frac{1}{m} T_{\max } \cos \alpha u_{t}-\dot{V}_{r}-\frac{e_{1} \dot{\tau}_{1}}{\tau_{1}}\right)
$$

Since both $S\left(v_{i}\right)$ and $\tau_{i}(t)$ are bounded functions, so $e_{i}(t)=\tau_{i}(t) S\left(v_{i}\right)$ is bounded. The bound of $S\left(v_{i}\right)$ is $\lim _{v_{i} \rightarrow+\infty} S\left(v_{i}\right)=\bar{\zeta}_{i}, \lim _{v_{i} \rightarrow-\infty} S\left(v_{i}\right)=-\zeta_{i}$, and $\tau_{i}(t)>0$, so $e_{i}(t)$ satisfies the predetermined performance $-\zeta_{i} \bar{\zeta}_{i} \tau_{i}(t)<e_{i}(t)<\bar{\zeta}_{i} \tau_{i}(t)$. Since $S\left(v_{i}\right)$ is an increasing function, if the control law is designed for the system (9) to make $v_{i}$ approach $0, S\left(v_{i}\right)$ is made to approach 0 . In addition, $\tau_{i}(t)$ is a smooth decreasing function, and $0<\lim _{t \rightarrow \infty} \tau_{i}(t)=\tau_{i \infty}<\tau_{i 0}$, so $e_{i}(t)$ can be made to approach 0 as $v_{i}$ approaches 0 .

\subsection{Design of Control Law Based on Backstepping Method}

After the transformation process in the above section 2.1, the design objective of the control law in this section changes from that the system output $y$ asymptotically tracks the reference signal $y_{r}$ (reference) to that the state of system (9) $\left[\begin{array}{ll}v_{1} & v_{2}\end{array}\right]^{T}$ asymptotically approaches zero.

As to system (9), there is

$\rho_{1}=1$ is the relative degree of the subsystem $e_{i}(t) \dot{v}_{1}=S_{1}\left(f_{1}+\frac{1}{m} T_{\max } \cos \alpha u_{t}-\dot{V}_{r}-\frac{e_{1} \dot{\tau}_{1}}{\tau_{1}}\right)$;

$\rho_{2}=2$ is the relative degree of the subsystem $\dot{v}_{2}=S_{2}\left(q-\dot{\theta}_{r}-\frac{e_{2} \dot{\tau}_{2}}{\tau_{2}}\right), \quad \dot{q}=f_{2}+\frac{1}{2 I_{y}} \rho V^{2} S \bar{c} C_{M}$.

The tracing error of the system is defined as

$$
z_{i j}\left(i=1,2 ; j=1, \cdots, \rho_{i}\right)
$$

To the two subsystems in Equation (9), the law of self-adaptive backstepping control is designed. The designing process of its control law is as follows:

In the first step, the control law is designed for the first subsystem.
If the ideal control law to be designed is defined as $u_{t c}^{0}$ and the actual control law $u_{t c}$, its influence on the speed tracking error $\eta_{11}$ will be adjusted and renovated according to the following adaptive law:

$$
\dot{\eta}_{11}=-c_{11} \eta_{11}+\frac{1}{m} T_{\max } \cos \alpha\left(u_{t c}-u_{t c}^{0}\right)
$$

In this formula, $c_{11}>0$ is the controller gain to be selected. According to Equation (8), $S_{1} \eta_{11}$, the influence of $u_{t c}$ on $u_{t c}^{0}$, can be obtained, and the tracking error after compensation is:

$$
\bar{z}_{11}=z_{11}-S_{1} \eta_{11}
$$

Take the derivative of formula (13), and it can be gotten:

$$
\begin{aligned}
\dot{\bar{z}}_{11}= & S_{1}\left(f_{1}+\frac{1}{m} T_{\max } \cos \alpha u_{t}-\dot{V}_{r}-\frac{e_{1} \dot{\tau}_{1}}{\tau_{1}}\right) \\
& +c_{11} S_{1} \eta_{11}-S_{1} \frac{1}{m} T_{\max } \cos \alpha\left(u_{t c}-u_{t c}^{0}\right)
\end{aligned}
$$

The Lyapunov function is designed as $V_{11}=\frac{1}{2} \bar{z}_{11}^{2}$. Obviously, because of $u_{t}=u_{t c}, V_{11} \geq 0$. In order to make $\dot{V}_{11}<0$, the expected control signal is designed as:

$$
u_{t c}^{0}=\frac{m}{T_{\max } \cos \alpha}\left(-\frac{c_{11} z_{11}}{S_{1}}+\dot{V}_{r}-f_{1}+\frac{e_{1} \dot{\tau}_{1}}{\tau_{1}}\right)
$$

In the second step, the control law is designed according to the second subsystem of (9).

1) Set $v_{2}$ as:

$$
z_{21}=v_{2}
$$

and take the derivative of formula (10), it can be gotten:

$$
\dot{z}_{21}=S_{2}\left(q-\dot{\theta}_{r}-\frac{e_{2} \dot{\tau}_{2}}{\tau_{2}}\right)
$$


If the ideal virtual control law to be designed is named as $q_{c}^{0}$ and the actually gained control law $q_{c}$, and its influence on the tracking error of the pitch angle is represented as $\eta_{21}$, the tracking compensation signal can be updated according to the following formula:

$$
\dot{\eta}_{21}=-c_{21} \eta_{21}+\left(q_{c}-q_{c}^{0}\right)
$$

In it, $c_{21}>0$ is the controller gain to be determined. According to formula (8), the influence of replacing $q_{c}^{0}$ with $q_{c}$ on $v_{2}$ is $S_{2} \eta_{21}$, and the tracking error after compensation is:

$$
\bar{z}_{21}=z_{21}-S_{2} \eta_{21}
$$

Take the derivative of formula (19), and it can be gotten:

$$
\dot{\bar{z}}_{21}=S_{2}\left(q-\dot{\theta}_{r}-\frac{e_{2} \dot{\tau}_{2}}{\tau_{2}}\right)+c_{21} S_{2} \eta_{21}-S_{2}\left(q_{c}-q_{c}^{0}\right)
$$

The expected control signal in this design is:

$$
q_{c}^{0}=-\frac{c_{21} z_{21}}{S_{2}}-\eta_{22}+\frac{e_{2} \dot{\tau}_{2}}{\tau_{2}}
$$

2) The tracing error of the pitch angle rate $q$ is defined as:

$$
z_{22}=q-\dot{\theta}_{r}-q_{c}
$$

Take the derivative of formula (22), and it can be gotten:

$$
\begin{aligned}
\dot{z}_{22}= & f_{2}+\left[q-\frac{1}{2 m} \rho V S\left(C_{l_{z}} \alpha+\frac{\bar{c}}{2 V} C_{D_{z}} q+\frac{\bar{c}}{2 V} C_{M_{z}} \dot{\alpha}\right)\right. \\
& \left.+\frac{1}{V} g(\sin \alpha \sin \theta+\cos \alpha \cos \theta)\right] u_{e}-\ddot{\theta}_{r}-\dot{q}_{c}
\end{aligned}
$$

If the ideal virtual control law to be designed is named as $u_{e c}^{0}$, and the influence of the actually gained control law $u_{e c}$ on the tracking error of the pitch angle is represented as $\eta_{22}$, the tracking compensation signal can be updated according to the following formula:

$$
\begin{aligned}
\eta_{22}= & -c_{22} \eta_{22}+\left[q-\frac{1}{2 m} \rho V S\left(C_{l_{z}} \alpha+\frac{\bar{c}}{2 V} C_{D_{z}} q+\frac{\bar{c}}{2 V} C_{M_{z}} \dot{\alpha}\right)\right. \\
& \left.+\frac{1}{V} g(\sin \alpha \sin \theta+\cos \alpha \cos \theta)\right]\left(u_{e c}-u_{e c}^{0}\right)
\end{aligned}
$$

In this formula, $c_{22}>0$ is the controller gain to be determined, and the tracking error after compensation is:

$$
\bar{z}_{22}=z_{22}-\eta_{22}
$$

Take the derivative of formula (25), and it can be gotten:

$$
\begin{aligned}
\dot{\bar{z}}_{22}= & f_{2}+\left[q-\frac{1}{2 m} \rho V S\left(C_{l_{z}} \alpha+\frac{\bar{c}}{2 V} C_{D_{z}} q+\frac{\bar{c}}{2 V} C_{M_{z}} \dot{\alpha}\right)\right. \\
& \left.+\frac{1}{V} g(\sin \alpha \sin \theta+\cos \alpha \cos \theta)\right] u_{e}-\ddot{\theta}_{r}-\dot{q}_{c}+c_{22} \eta_{22} \\
& -\left[q-\frac{1}{2 m} \rho V S\left(C_{l_{z}} \alpha+\frac{\bar{c}}{2 V} C_{D_{z}} q+\frac{\bar{c}}{2 V} C_{M_{z}} \dot{\alpha}\right)\right. \\
& \left.+\frac{1}{V} g(\sin \alpha \sin \theta+\cos \alpha \cos \theta)\right]\left(u_{e c}-u_{e c}^{0}\right)
\end{aligned}
$$

The Lyapunov function is designed as $V_{22}=\frac{1}{2} \bar{z}_{21}^{2}+\frac{1}{2} \bar{z}_{22}^{2}$. Obviously, $V_{22} \geq 0, V_{11} \geq 0$. In order to make $\dot{V}_{22}<0$, the expected control signal is designed as:

$$
u_{e c}^{0}=\frac{\left(-c_{22} z_{22}-S_{2} \bar{z}_{21}-f_{2}+\dot{q}_{c}+\ddot{\theta}_{r}\right)}{q-\frac{1}{2 m} \rho V S\left(C_{l_{z}} \alpha+\frac{\bar{c}}{2 V} C_{D_{z}} q+\frac{\bar{c}}{2 V} C_{M_{z}} \dot{\alpha}\right)+\frac{1}{V} g(\sin \alpha \sin \theta+\cos \alpha \cos \theta)}
$$

So far, the expected control law $\left[\begin{array}{lll}u_{t c}^{0} & q_{c}^{0} & u_{e c}^{0}\end{array}\right]^{T}$ is obtained in formula (15), (21) and (27).

\subsection{Design of Control Allocation Algorithm for Multiple Rudder Surface}

The control surface of the flying-wing UAV has a high redundancy configuration. The expected virtual control law should be assigned to each rudder surface through control allocation. Since only two pairs of elevons are considered in the longitudinal control, the principle of the allocation of the rudder surfaces is as follows:

1) The deflection angles of the four rudder surfaces meet the requirements of pitching moment. In terms of the flying-wing UAV studied in this chapter, the steerage of the four rudder surfaces in pitch control is the same, so it is required that the sum of the deflection angles of the four rudder surfaces is equal to the expected deflection angle of the elevator $u_{e c}^{0}$;

2) The sum of the rolling moment caused by the deflection angles of the four rudder surfaces is zero. The sum of the deflection angles of the two left rudder surfaces of the sample UAV is equal to the sum of the deflection angles of the two right rudder surfaces.

3) The deflection angles of the two rudder surfaces on the same side are distributed according to the proportion of the maximum achievable deflection angle. In this paper, the maximum achievable deflection angles of the elevons of the aircraft are the same, so the deflection angles of the two rudder surfaces on the same side can be evenly distributed.

When the control surface is in malfunction, the fault compensation shall be carried out under the premise of following the above principle of the rudder surface 
allocation. Set the control law of the system as $u=\left[\begin{array}{llllll}u_{t c 1} & u_{t c 2} & u_{c 1} & u_{c 1}^{\prime} & u_{c 2} & u_{c 2}^{\prime}\end{array}\right]^{T}$ and the corresponding ideal control quantity as $u=\left[\begin{array}{llllll}u_{t c 1}^{0} & u_{t c 2}^{0} & u_{c 1}^{0} & u_{c 1}^{\prime 0} & u_{c 2}^{0} & u_{c 2}^{\prime 0}\end{array}\right]^{T}$. Considering the malfunction of the elevator as shown in formular (2), $u_{e c}^{0}\left[\begin{array}{lllll}u_{c 1}^{0} & u_{c 1}^{\prime 0} & u_{c 2}^{0} & u_{c 2}^{\prime 0}\end{array}\right]^{T}$ are respectively the sum of the output of each expected elevator, and $\left[\begin{array}{llll}u_{c 1}^{0} & u_{c 1}^{\prime 0} & u_{c 2}^{0} & u_{c 2}^{\prime 0}\end{array}\right]^{T}$ are respectively the input of each expected elevator. If $K_{1}=\operatorname{diag}\left\{\lambda_{1} \lambda_{2} \lambda_{3} \lambda_{4}\right\}$ and $K_{1 i}=\lambda_{i}, i=1,2,3,4 \quad, \quad K_{2}=\bar{u}_{e}=\left[\begin{array}{llll}\bar{u}_{1} & \vec{u}_{1} & \bar{u}_{2} & \vec{u}_{2}^{\prime}\end{array}\right]^{T}$ $N_{1} \in \square^{4 \times 1}(i=1,2,3,4)$ is a column vector whose number $i$ element is 1 and other elements are 0 . The specific design steps are as follows:

Step 1, according to distribution principle (1), it can be obtained:

$$
\sum_{i=1}^{4} K_{1 i} u_{c 1}^{0}+N_{1}^{T} K_{2}=\sum_{i=1}^{4} \lambda_{1} u_{c 1}^{0}+\bar{u}_{1}=u_{e c}^{0}
$$

Step 2, according to distribution principle (2), it can be obtained:

$$
\lambda_{1} u_{c 1}^{0}+\bar{u}_{1}+\lambda_{3} u_{c 2}^{0}+\bar{u}_{2}=\lambda_{2} u_{c 1}^{\prime 0}+\vec{u}_{1}^{\prime}+\lambda_{4} u_{c 2}^{\prime 0}+\vec{u}_{2}^{\prime}
$$

After processed through formular (5.2), it can be obtained:

$$
\begin{aligned}
& u_{e c}^{0}=2\left(K_{11} u_{c 1}^{0}+K_{13} u_{c 2}^{0}+\left(N_{1}+N_{3}\right)^{T} K_{2}\right) \\
& u_{e c}^{0}=2\left(K_{12} u_{c 1}^{\prime 0}+K_{14} u_{c 2}^{\prime 0}+\left(N_{2}+N_{4}\right)^{T} K_{2}\right)
\end{aligned}
$$

Step 3, according to distribution principle (3), the distribution rate of rudder surface can be obtained:

$$
u_{c 1}^{0}=\left\{\begin{array}{c}
0, \\
\widehat{K}_{1 i}=0, i=1,2,3,4 \\
\frac{1}{2 \widehat{K}_{1 i}}\left[\frac{1}{2} u_{e c}^{0}-\left(N_{1}+N_{3}\right)^{T} \widehat{K}_{2}\right], i=1,3 \\
\frac{1}{2 \widehat{K}_{1 i}}\left[\frac{1}{2} u_{e c}^{0}-\left(N_{2}+N_{4}\right)^{T} \widehat{K}_{2}\right], i=2,4, \widehat{K}_{1 i} \neq 0 \\
u_{t c 1}^{0}=u_{t c 2}^{0}=\frac{1}{2} u_{t c}^{0}
\end{array}\right.
$$

$\widehat{K}_{1 i}$ and $\hat{K}_{2}$ in formula (32) will be given in the next section.

\subsection{Design of the adaptive adjustment law}

For in the Lyapunov function $\dot{V}_{22}$ :

$$
\begin{aligned}
& u_{e}=\sum_{i=1}^{4} K_{1 i} u_{c 1}+\left(N_{1}+N_{2}+N_{3}+N_{4}\right)^{T} K_{2} \\
& u_{e c}=\sum_{i=1}^{4} \widehat{K}_{1 i} u_{c 1}+\left(N_{1}+N_{2}+N_{3}+N_{4}\right)^{T} \widehat{K}_{2}
\end{aligned}
$$

if $\tilde{K}_{1}=\widehat{K}_{1}-K_{1}, \tilde{K}_{2}=\widehat{K}_{2}-K_{2}$, it can be obtained that:

$$
u_{e c}-u_{e}=\sum_{i=1}^{4} \tilde{K}_{1 i} u_{c 1}+\left(N_{1}+N_{2}+N_{3}+N_{4}\right)^{T} \tilde{K}_{2}
$$

If the Lyapunov function is designed as:

$$
V_{L}(t)=\frac{1}{2} \bar{z}^{T} \bar{z}+\frac{1}{2} \operatorname{tr}\left[\tilde{K}_{1}^{T} \Lambda_{1} \tilde{K}_{1}\right]+\frac{1}{2} \tilde{K}_{2}^{T} \Lambda_{2} \tilde{K}_{2}
$$

in it:

$\bar{z}=\left[\begin{array}{lll}\bar{z}_{11} & \bar{z}_{21} & \bar{z}_{22}\end{array}\right]^{T}, \Lambda_{1}=\operatorname{diag}\left\{\begin{array}{llll}\Lambda_{11} & \Lambda_{12} & \Lambda_{13} & \Lambda_{14}\end{array}\right\} \in \square^{4 \times 4}>0, \Lambda_{2} \in \square^{4 \times 4}>0$ is the

positive definite diagonal matrix to be selected.

In order to make $\dot{V}_{L}(t)<0$, the adaptive adjustment law of the fault parameter is designed as:

$$
\begin{aligned}
\dot{\bar{K}}_{1 i}= & \frac{1}{\Lambda_{1 i}} \bar{z}_{22}\left[q-\frac{1}{2 m} \rho V S\left(C_{l_{z}} \alpha+\frac{\bar{c}}{2 V} C_{D_{z}} q+\frac{\bar{c}}{2 V} C_{M_{z}} \dot{\alpha}\right)\right. \\
& \left.+\frac{1}{V} g(\sin \alpha \sin \theta+\cos \alpha \cos \theta)\right] u_{c 1} \\
\dot{\bar{K}}_{2}= & \Lambda_{2}^{-1} \bar{z}_{22}\left[q-\frac{1}{2 m} \rho V S\left(C_{l_{z}} \alpha+\frac{\bar{c}}{2 V} C_{D_{z}} q+\frac{\bar{c}}{2 V} C_{M_{z}} \dot{\alpha}\right)\right. \\
& \left.+\frac{1}{V} g(\sin \alpha \sin \theta+\cos \alpha \cos \theta)\right]\left(N_{1}+N_{2}+N_{3}+N_{4}\right)
\end{aligned}
$$

\subsection{Analysis of System Stability}

According to the design process of the above control law, under the action of the control law (15), (21), (27) and the adaptive adjustment law of the parameter (36), the the derivative of the tracking compensation error is:

$$
\begin{aligned}
& \dot{\bar{z}}_{11}=-c_{11} \bar{z}_{11} \\
& \dot{\bar{z}}_{21}=-c_{21} \bar{z}_{21}+S_{2} \bar{z}_{22} \\
& \left.\dot{\bar{z}}_{22}=-c_{22} \bar{z}_{22}-S_{2} \bar{z}_{21}-f_{3} \llbracket \sum_{i=1}^{4} \tilde{K}_{1 i} u_{c 1}+\left(N_{1}+N_{2}+N_{3}+N_{4}\right)^{T} \tilde{K}_{2}\right]
\end{aligned}
$$

In it:

$$
f_{3}=q-\frac{1}{2 m} \rho V S\left(C_{l_{z}} \alpha+\frac{\bar{c}}{2 V} C_{D_{z}} q+\frac{\bar{c}}{2 V} C_{M_{z}} \dot{\alpha}\right)+\frac{1}{V} g(\sin \alpha \sin \theta+\cos \alpha \cos \theta)
$$

and then the derivative of the selected Lyapunov function is:

$$
\begin{aligned}
\dot{V}_{L}(t) & =\sum_{i=1}^{4}\left[\tilde{K}_{1 i}\left(-\bar{z}_{22} f_{3} u_{c 1}+\Lambda_{1 i} \dot{\tilde{K}}_{1 i}\right)\right] \\
& -\sum_{i=1} \sum_{j=1} c_{i j} \bar{z}_{i j}^{2}+\left(\dot{\tilde{K}}_{2}^{T} \Lambda_{2}-\bar{z}_{22} f_{3}\left(N_{1}+N_{2}+N_{3}+N_{4}\right)^{T}\right) \tilde{K}_{2}(38) \\
& =\sum_{i=1} \sum_{j=1} c_{i j} \bar{z}_{i j}^{2} \leq 0
\end{aligned}
$$


Therefore, the system is stable. In this paper, $V_{r}, \theta_{r}$ and its derivative of each order is set to be bounded, and then $\tilde{z}_{i j}, \tilde{K}_{1}, \tilde{K}_{2}$ is bounded and $V_{L}(t)$ is also bounded, so that:

$$
\int_{0}^{\infty} \sum_{i=1} \sum_{j=1} \bar{z}_{i j}^{2} d t \leq-\frac{1}{c} \int_{0}^{\infty} \dot{V}_{L}(t) d t \leq-\frac{1}{c}\left(V_{L}(\infty)-V_{L}(0)\right)<\infty
$$

From formula (5.36), it can be obtained that $\dot{\bar{z}}_{i j}$ exists and is bounded. According to the barbalat's lemma,

$$
\lim _{t \rightarrow \infty} \bar{z}_{i j}=0
$$

Therefore, formula (9) of the system is asymptotically stable, and the tracking compensation error asymptotically approaches 0 . This also means that when the control surface is not saturated, $\bar{z}_{i j}$ converges to $z_{i j}$; in the saturation stage of the control surface, the actual tracking error $z_{i j}$ will increase, but the tracking compensation error still converges and the error estimation process is still stable. Furthermore, according to the bounded transformation of the system in section 1.1 , the velocity and pitch angle of system (1) can asymptotically track the given reference signal, and the tracking error is within the predetermined range of performance bounds.

Therefore, for system (9), the control law of backstepping compensation (15), (21), (27) and the parameter self-adaptive adjustment law (36), as well as the distribution law of the rudder surface control (31) and (32), are designed, so that all states of the system can be bounded and the tracking compensation error asymptotically approaches 0 .

\section{MATLAB / Simulink Simulation Results}

The flying-wing UAV researched in this paper is put through the trim calculation at a cruising altitude of 11,000 meters and a cruising speed of Mach 0.6. In the trimming result, the angle of attack is $4^{\circ}$, the pitch angle is $4^{\circ}$, the sum of throttle openings is 0.3041 , and the sum of the deflection angles of the elevators is $-12.1742^{\circ}$. Take $u_{t 1}$ and $u_{t 2}$ as the variables of the throttle opening, and we'll get $u_{t}=u_{t 1}+u_{t 2}+0.3041$. After the trimming calculation, the pitch angle of the UVA's elevator is zero, namely, $u_{e}=u_{1}+u_{1}^{\prime}+u_{2}+u_{2}^{\prime}$.

The signal of reference velocity is $V_{r}=130+20 \cos (0.1 t)$; the signal of reference pitch angle is $\theta_{r}=18+10 \sin (0.1 t)$. Considering such factors as the limitation in the UAV's flight characteristics, the actuator's malfunction and the actuator saturation constraint, the boundary-limited transformation parameters of the velocity tracking are designed as:

$$
\tau_{1}(t)=48.7 e^{-0.2 t}+1.3,-\zeta_{1}=0.5, \bar{\zeta}_{1}=1
$$

and the transformation parameters of pitch angle tracking as:

$$
\tau_{2}(t)=7.7 e^{-0.2 t}+0.3,-\zeta_{2}=0.8, \bar{\zeta}_{2}=1
$$

When the UVA's malfunction is set as $80 \mathrm{~s}$, a $60 \%$ malfunction of losing effectiveness will occur in the left internal elevator $u_{1}$, and the simulation result is obtained finally, as shown in the following figure.

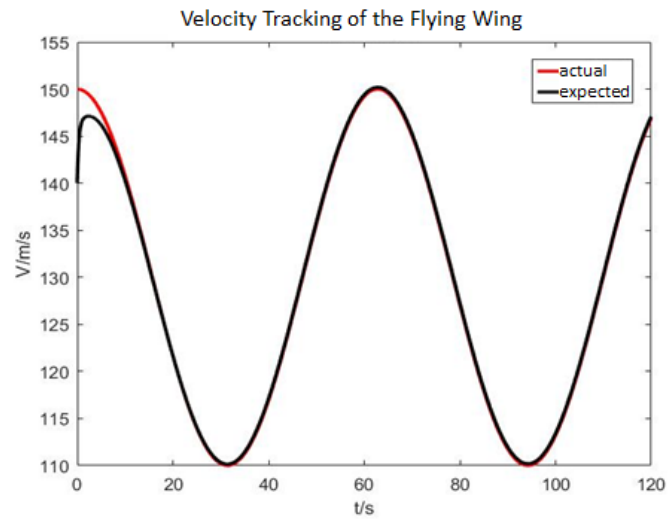

Figure 2 The Velocity Tracking Curve of the Flying-Wing UAV

It can be seen from Figure 2 that the system has a good tracking performance for velocity. Although there is a big gap between the initial speed and the reference speed, the system can track the reference speed signal from second 9.5 . 


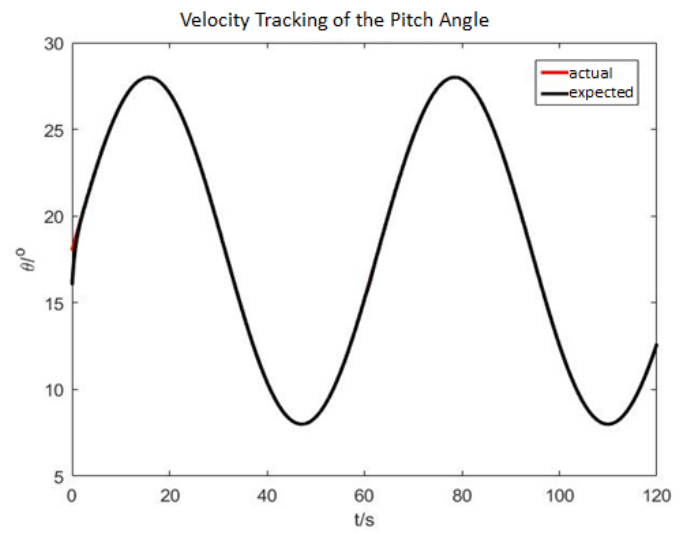

Figure 3 The Pitch Angle Tracking Curve of the Flying-Wing UAV

It can be seen from Figure 4 that the pitch angle fluctuates slightly in the initial stage, but it can track the signal of the reference pitch angle well all the time. The simulation results show that the system plays an improving role obviously in the phases of dynamic adjustment and steady maintenance.

Figure 4 and Figure 5 show the tracking error of velocity and the tracking error of pitch angle under the self-adaptive backstepping fault-tolerant control law. It can be seen from the figure that the tracking error of the system can be effectively constrained within the predetermined performance limit by the transformation method of limiting performance, so that the steady-state error and overshoot of the system can be effectively controlled and better system performance can be obtained.

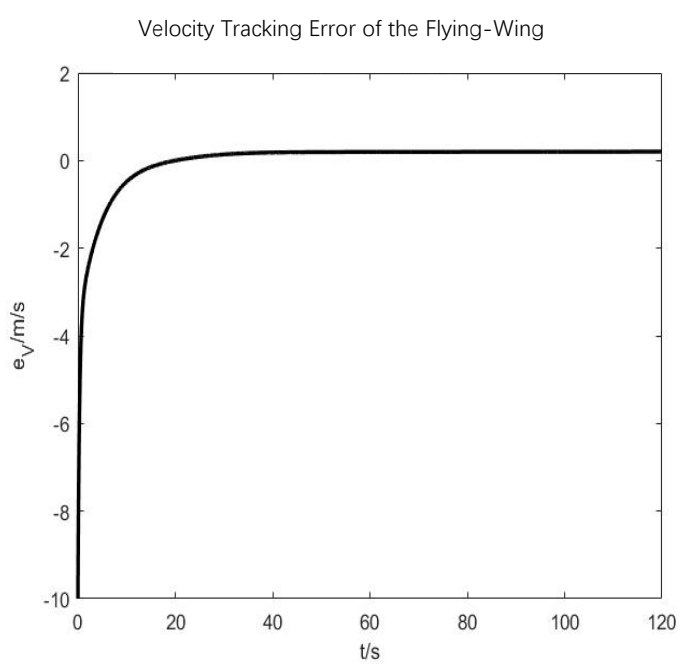

Figure 4 The Velocity Tracking Error of the Flying-Wing UAV

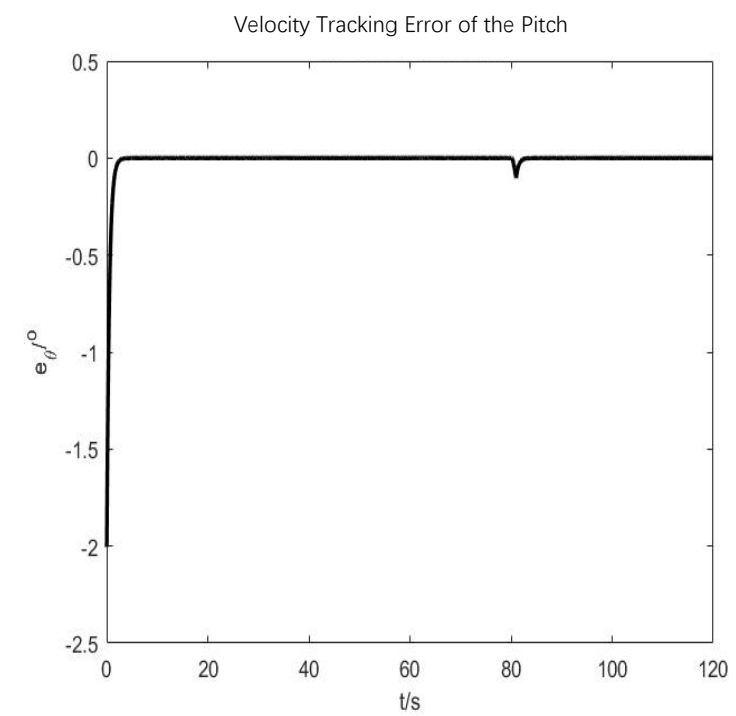

Figure 5 The Pitch Angle Tracking Error of the Flying-Wing UAV 
Figure 6 show the input signals of the flying-wing UAV, Figure 6 is the variant of the deflection angle of the elevator. It can be seen from the figure that in the 80 $\mathrm{s}$ when the malfunction of losing effectiveness occurs in elevator $u_{1}$, the deflection angles of other rudder surfaces increase rapidly. At this moment, the control allocation will be reperformed in the system, and the redundant actuator will immediately action to compensate the impact from the malfunction on the overall deflection angles of the elevators. Therefore, there is no obvious fluctuation in the speed, angle of attack, pitch angle or pitching angle rate under the influence of the malfunction. Moreover, the deflection angle of the elevator also reaches saturation during the initial period, but due to the transformation law of the preceding amplitude limiting, the instruction for the deflection angle of elevator won't exceed its amplitude limit. Therefore, for the actual flying-wing UAV, under the control shown in Figure 6, the tracking effect shown in Figure 2-3 can be achieved. If the limiting transformation is not carried out, the control signals needed to track a given signal may exceed the physical limits of the actuator. In the real UAV flight, it may not be able to provide the required control signals, which will affect the tracking effect and even lead to the system instability.

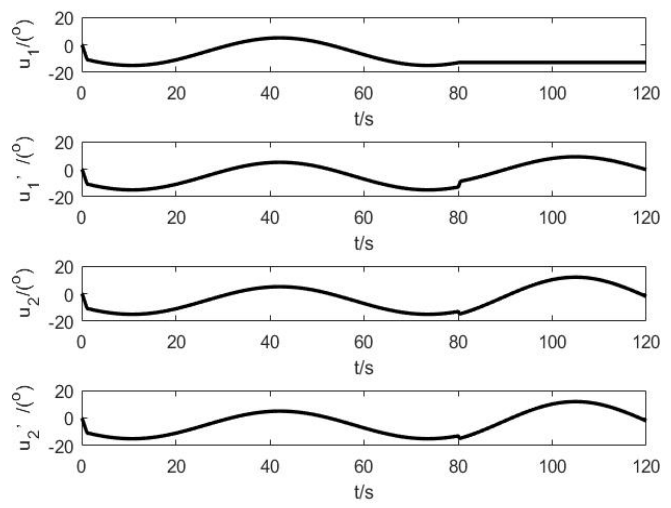

Figure 6 The variation Curve of the Deflection Angle of the Elevator Rudder Surface of the Flying-Wing UAV

\section{Conclusion}

This chapter puts forward the self-adaptive backstepping fault-tolerant control method for the flying-wing UAV. It considers the conditions for the partial malfunction of the rudder surface of lifting function and the saturation characteristics of the rudder surface and the throttle in the longitudinal nonlinear system model. With the PPB transformation method, the output error is constrained effectively, the tracking problem is successfully transformed into the stabilization problem, and the system is forced to be endowed with the good dynamic performance limitations. The adaptive compensation method is adopted to design the dynamic regulation parameters, and the control allocation strategy based on backstepping method is designed in combination with the characteristics of the flying-wing UAV. From the final simulation results, it can be seen that the control law designed in this paper can not only guarantee the closed-loop stability of the system when the malfunction of the rudder surface occurs, but also asymptotically track the given reference signal, and constrain the tracking error within the predetermined performance bound.

To sum up, the nonlinear fault-tolerant control of the flying-wing UAV based on control allocation, designed in this paper, achieves the expected control effect, and the system has a good fault-tolerant performance.

\section{References}

1. Li YB, Li Z, Zhang XD. Research status and development of control methods for UAV flight. Flight Dynamics. 2011 Apr 15; 61-1172: 1-5+9.

2. Zhang P, Wang J. A research on the control law of the longitudinal posture of the small-size fixed-wing UAV. Computer Measurement \& Control. 2015 Aug. 25; 11-4762: 2686-2688.

3. Zhang BQ, Ma Y, Chu HB. A study on the combined rudder surfaces with the course control of the low-aspect-ratio flying wing. Acta Aeronautica et Astronautica Sinica. 2013 Mar. 25; 11-1929: 2435-2442.

4. Tan J, Zhou Zh, Zhu XP, Zhang L. Posture control of the fractional integral sliding framework of the flying-wing UAV. Control Theory \& Applications. 2015 Jul. 29; 44-1240: 607-614.

5. Yu J, Chen M, Jiang, CS. Adaptive sliding model control for nonlinear uncertain systems based on disturbance observer. Control Theory \& Applications. 2014 Aug. 31: 993-999.

6. ROSE B. Flying Wings and Tailless Aircraft [M]. Hinckley, UK: Midland, 2010.

7. SAEED T I, GRAHAM W R, BABINSKY H, et al. Conceptual design for a laminar-flying-wing aircraft [D]. London, UK: Cambridge University, 2012.

8. BENOSMAN M, LUM K Y. Passive actuators' fault-tolerant control for affine nonlinear systems [J]. 
IEEE Transactionson Control Systems Technology, 2009, 18(1): $152-163$.

9. DING Z. Nonlinear and Adaptive Control Systems [M]. London: IET, 2013.

10. SWAROOP D, HEDRICK J, YIP P, et al. Dynamic surface control for a class of nonlinear systems [J]. IEEE Transactions on Automatic Control, 2002, 45(10): 1893 - 1899 .

11. QIU Xiangwei, ZHANG Shaojie, LIU Chunsheng. Adaptive compensation control considering the transient performance of a class of MIMO nonlinear systems with actuator failures [J]. Information and Control, 2014, 43(1): 63 - 67. 